\title{
Polymorphisms in Genes of the De Novo Lipogenesis Pathway and Overall Survival of Hepatocellular Carcinoma Patients Undergoing Transarterial Chemoembolization
}

\author{
You-Sheng Wu' ${ }^{1 \&}$, Deng-Ke Bao ${ }^{1,2 \&}$, Jing-Yao Dai ${ }^{3}$, Cheng Chen ${ }^{1}$, Hong-Xin \\ Zhang $^{4}$, YeFa Yang ${ }^{5}$, Jin-Liang Xing ${ }^{1}$, Xiao-Jun Huang ${ }^{1 *}$, Shao-Gui Wan ${ }^{2 *}$
}

\begin{abstract}
Aberrant expression of genes in de novo lipogenesis (DNL) pathway were associated with various cancers, including hepatocellular carcinoma (HCC). Single nucleotide polymorphisms (SNPs) of DNL genes have been reported to be associated with prognosis of some malignancies. However, the effects of SNPs in DNL genes on overall survival of HCC patients receiving transarterial chemoembolization (TACE) treatment are still unknown. In present study, nine SNPs in three genes (ACLY, ACACA and FASN) in DNL pathway were genotyped using the Sequenom iPLEX genotyping system in a hospital-based cohort with 419 HCC patients treated with TACE, and their associations with HCC overall survival were evaluated by Cox proportional hazard regression analysis under three genetic models (additive, dominant and recessive). Although we did not find any significant results in total analysis (all $p>0.05$ ), our stratified data showed that SNP rs9912300 in ACLY gene was significantly associated with overall survival of HCC patients with lower AFP level and SNP rs11871275 in ACACA gene was significantly associated with overall survival of $\mathrm{HCC}$ patients with higher AFP level. We further identified the significant interactions between AFP level and SNP rs9912300 or rs11871275 in the joint analysis. Conclusively, our data suggest that genetic variations in genes of DNL pathway may be a potential biomarker for predicting clinical outcome of HCC patients treated with TACE.
\end{abstract}

Keywords: De novo lipogenesis - single nucleotide polymorphism - hepatocellular carcinoma - overall survival - TACE

Asian Pac J Cancer Prev, 16 (3), 1051-1056

\section{Introduction}

Hepatocellular carcinoma (HCC) is one of the most prevalent malignant tumors and leading cause of cancerrelated deaths worldwide (Jemal et al., 2010). In recent years, the morbidity and mortality rates of HCC have escalated, particularly in China (Jemal et al., 2011). Despite improvements in surveillance and clinical treatment strategies, there are only 10 to $20 \%$ of HCC can be surgically excised (Lin et al., 1997). Most HCC patients are diagnosed at intermediate to advanced stages, and thus curative therapies such as resection, transplantation, or percutaneous ablation are not suitable(Bruix and Sherman, 2011). As the most widely-used treatment for unresectable HCC, transarterial chemoembolization (TACE) is recommended as the first-line therapy for HCC patients at intermediate stage (Llovet et al., 2002; Llovet and Bruix, 2003). However, the prognosis of HCC patients treated by TACE is greatly diverse according to disease status. Therefore, the discovery of biomarkers for predicting HCC prognosis after TACE treatment is urgently wanted.
One of the most important cancer hallmarks is the metabolic reprogramming in cancerous cells, which includes the increased de novo lipogenesis (DNL) in spite of extracellular lipids levels (Menendez and Lupu, 2007; Abramson, 2011). Three metabolic rate-limiting enzymes, which are ATP citrate lyase (ACLY), acetyl-CoA carboxylase 1 (ACACA) and fatty-acid synthase (FASN), consist of the core DNL pathway. ACLY is a cytosolic enzyme involved in the first step of DNL, and catalyzes citrate into acetyl-CoA, which is a vital building block for the endogenous biosynthesis of fatty acids, isoprenoid and cholesterol (Watson et al., 1969; Hatzivassiliou et al., 2005; Zaidi et al., 2012a; Zaidi et al., 2012b). It has been reported that ACLY is highly upregulated in several cancers, including cancers of breast, liver, brain, colon, lung, stomach, bladder, and prostate (Zaidi et al., 2012b). ACACA is an enzyme which locates in endoplasmic reticulum and converts acetyl-CoA to malonyl-CoA (Yoon et al., 2007). Deletion of ACACA in hepatocytes reduces the expression levels of malonyl-CoA and de novo lipid synthesis (Mao et al., 2006). FASN is a key

${ }^{1}$ State Key Laboratory of Cancer Biology, Experimental Teaching Center of Basic Medicine, ${ }^{3}$ Department of Hepatobiliary Surgery, Xijing Hospital, ${ }^{4}$ Department of Pain treatment, Tangdu Hospital, Fourth Military Medical University, Xi'an, Shaanxi, ${ }^{2}$ Institute of Pharmacy, Pharmaceutical College of Henan University, Kaifeng, ${ }^{5}$ Department of Interventional Radiology, Eastern Hepatobiliary Surgery Hospital, Second Military Medical University, Shanghai, China ${ }^{\circledR}$ Equal contributors *For correspondence: wansg@henu.edu.cn 
enzyme involved in the last step of fatty acid production, in which long chain fatty acid is synthesizd by using acetyl-CoA and malonyl-CoA as substrates (Menendez and Lupu, 2007; Dorn et al., 2010). It has reported that FASN is highly expressed in many cancers, including cancers of prostate, breast, lung, ovary, bladder, stomach, oral cavity and melanoma (Swinnen et al., 2006; Migita et al., 2009). Accumulating evidences have suggested that aberrant glycolysis and lipid synthesis contribute to cancer progression, and would be potential therapeutic targets (Mashima et al., 2009; Migita et al., 2009; Furuta et al., 2010). A recent study suggested that suppression of genes ACLY, ACACA, and FASN, which are involved in DNL, has induced the proliferation and survival of HCC cell lines (Calvisi et al., 2011).

Single nucleotide polymorphism (SNP) is the most common type of genetic variation, which can be used as a biomarker of genetic background to predict the risk, therapeutic response and prognosis of malignancies (Laing et al., 2011; Liu et al., 2012). Excepting for amino acid change, SNPs directly affect gene functions through various translational or post-translational mechanisms, such as altering miRNA binding, gene splicing, protein folding or mRNA degradation(Chamary et al., 2006). There were several studies have investigated the association between polymorphisms of DNL genes and development or prognosis of cancers (Nguyen et al., 2010; Eggert et al., 2012). Previously, our group has identified that SNPs in ACLY and FASN genes are significantly associated with clinical outcomes in non-small cell lung cancer patients (Jin et al., 2014). However, up to date, few studies have focused on the relationship between polymorphisms of ACLY, ACACA or FASN and the prognosis of HCC patients treated by TACE. In this study, we sought to assess the associations between functional polymorphisms in DNL pathway genes and overall survival in a hospital-based Chinese population diagnosed with HCC and treated by TACE.

\section{Materials and Methods}

\section{Study population and data collection}

A total of 448 Han Chinese patients with unresectable HCC were recruited at the Department of Radioactive Intervention of Eastern Hepatobiliary Surgery Hospital, Second Military Medical University in Shanghai, China between January 2008 and November 2011. All patients had no previous history of other cancers or cancer-related treatment at enrollment, and were newly diagnosed as HCC by imaging technologies and laboratory tests. TACE was used as the first-line treatment for all patients. TACE treatment was applied as previously described (Yuan et al., 2014). Among them, 29 cases with incomplete clinical information or follow-up data or poor DNA quality were excluded. Finally, 419 patients were successfully genotyped and included in primary cohort for further analysis. Detailed clinical information was collected through medical chart review by treating physicians. And standard follow-up was performed by a trained clinical specialist through on-site interview, direct calling, or medical chart review. The latest follow-up data in this analysis were obtained in July 2013. Overall survival time was defined as the interval from the first TACE treatment to the date of death or last follow-up. Patients who were lost during the follow-up were censored for the analysis. This study was approved by the Ethic Committee of the Second Military Medical University, and signed informed consent was obtained from each participant.

\section{SNP genotyping}

Five milliliter of venous blood sample was collected from each patient, and genomic DNA was isolated from whole blood using the E.Z.N.A. Blood DNA Midi Kit (Omega Bio-Tek, Norcross, GA) as described previously (Xing et al., 2011). The candidate SNPs in three DNL pathway genes (ACLY, ACACA, and FASN) were selected using a set of web-based SNP selection tools (http://snpinfo.niehs.nih.gov/snpinfo/snpfunc.htm) as described previously (Zhou et al., 2012). In general, the criteria and strategy for the selection of tagger SNPs were as follows: a) located in miRNA binding sites of the 3'untranslated region (3-UTR), in the transcription factor binding site of 5'flanking region (2,000 bp upstream from the transcription start site), in the mRNA splice site, or missense SNPs in exons; b) had potentially functions predicted by SNP selection tools; c) with minor allele frequency (MAF) more than $>5 \%$ in Han Chinese population (CHB). If there were multiple candidate SNPs within the same block and the linkage coefficient $\mathrm{r} 2>0.8$, only one SNP will be included. Based on the above criteria, nine candidate SNPs in three genes of DNL pathway were selected in this study. Genotyping was carried out on the iPLEX genotyping system (Sequenom, San Diego, CA). Laboratory persons who conducted genotyping analysis were blinded to patients' information. Strict quality controls were implemented during genotyping with more than $99.0 \%$ concordance in samples. Samples did not follow the quality controls were repeated once and those were omitted if unqualified again.

\section{Statistical analysis}

The SPSS version 19.0 software package (IBM, Armonk, NY, USA) was used for the analyses. Three genetic models (additive, dominant and recessive) were used to evaluate the association of single SNP with overall survival of HCC patients by Cox proportional hazard model. The best-fitting model was defined as that with the smallest $\mathrm{P}$ value and a lower trend $\mathrm{P}$ value. To exclude the effects of confounding factors, the association between SNPs in DNL genes and overall survival of HCC was assessed in three genetic models stratified by host characteristics using multivariate analysis. Kaplan-Meier curves were used to distinguish subgroup patients who had different overall survival outcome. All $\mathrm{P}$ values in this study were two-sided, and $p<0.05$ was considered to be significant.

\section{Results}

Characteristics of the study population and prognosis analysis on $\mathrm{HCC}$

Among the total of $419 \mathrm{HCC}$ patients with first-line 
TACE treatment included in the primary cohort, there were 326 patients died during the median follow-up time of 9.9 months. Majority of the patients were males $(87.4 \%)$ and with positive serum HBsAg $(85.7 \%)$. There were more patients with younger age $(53 \%)$, with late stage disease $(52 \%)$, and with higher level of serum AFP $(54.9 \%)$. Some patients $(18.6 \%)$ received others drug treatments other than TACE as first line treatment protocol (Table 1). The result of multivariate analyses by Cox proportional hazard regression model showed that there is a significant poor overall survival in patients with later TNM stage disease $(\mathrm{HR}=1.87,95 \%$ CI $1.48-2.35, p<0.001)$, in patients with higher level of serum AFP $(\mathrm{HR},=1.40,95 \%$ CI 1.11-1.77, $p=0.004)$. Significant improved overall survival was observed in patients received treatments other than TACE with HR of 0.40 (95\%CI 0.29-0.56, $p<0.001)$, comparing to those received TACE only (Table 1).

Association between SNPs in DNL pathway related genes and overall survival of HCC patients treated by TACE

We assessed the association between nine SNPs in three DNL pathway related genes (ACLY, ACACA and FASN) and the overall survival of HCC patients treated with TACE by Cox proportional hazard regression model using multivariate analysis under dominant, recessive, and additive genetic models. The results were presented as best-fit model. As the data shown in Table 2, there was no significant association between individual SNP and the overall survival of HCC patients. To exclude the potential effects of confounding factors, we then conducted the stratified analysis by gender, age, serum HBsAg status, TNM stage, serum AFP level, and treatment. Interestingly, SNP rs9912300 in ACLY gene exhibited significant associations with overall survival in HCC patients with lower serum $\mathrm{AFP}(<200 \mathrm{ng} / \mathrm{ml})$ under additive $(\mathrm{HR}=1.38$,

Table 1. Distribution of Patients' Characteristics and Prognosis Analysis

\begin{tabular}{|c|c|c|c|c|}
\hline $\begin{array}{r}\text { Parameter } \\
\text { a }\end{array}$ & $\begin{array}{l}\text { Number of } \\
\text { ll patients }(\%)\end{array}$ & $\begin{array}{l}\text { Number o } \\
\text { death (\%) }\end{array}$ & $\begin{array}{cc}\mathrm{HR} \\
(95 \% \mathrm{CI})^{*}\end{array}$ & $\overline{p \text { value }}$ \\
\hline \multicolumn{5}{|l|}{$\overline{\text { Gender }}$} \\
\hline Female & $53(12.6)$ & $40(12.3)$ & Ref. & \\
\hline Male & $366(87.4)$ & $286(87.7)$ & $1.12(0.80-1.57)$ & 0.499 \\
\hline \multicolumn{5}{|l|}{ Age, years } \\
\hline$<60$ & $222(53.0)$ & $172(52.8)$ & Ref. & \\
\hline$\geq 60$ & $197(47.0)$ & $154(47.2)$ & $0.99(0.79-1.25)$ & 0.956 \\
\hline \multicolumn{5}{|l|}{$\mathrm{HBs} A g$} \\
\hline Negative & $60(14.3)$ & $47(14.4)$ & Ref. & \\
\hline Positive & $359(85.7)$ & $279(85.6)$ & $0.81(0.59-1.13)$ & 0.220 \\
\hline \multicolumn{5}{|l|}{ TNM stage } \\
\hline I-II & 201(48.0) & $134(41.1)$ & Ref. & \\
\hline III-IV & $218(52.0)$ & $192(58.9)$ & $1.87(1.48-2.35)$ & $<0.001$ \\
\hline \multicolumn{5}{|c|}{ Serum AFP $(\mathrm{ng} / \mathrm{ml})$} \\
\hline$<200$ & $189(45.1)$ & $136(41.7)$ & Ref. & \\
\hline$\geq 200$ & $230(54.9)$ & $190(58.3)$ & $1.40(1.11-1.77)$ & 0.004 \\
\hline \multicolumn{5}{|l|}{ Treatment } \\
\hline TACE & $341(81.4)$ & $284(87.1)$ & Ref. & \\
\hline TACE+oth & ers $78(18.6)$ & $42(12.9)$ & $0.40(0.29-0.56)$ & $<0.001$ \\
\hline
\end{tabular}

Abbreviations: CI, confidence interval; HR, hazard ratio; *Adjusted for gender, age, HBsAg, serum AFP level, TNM stage, and treatment after surgery, where appropriate; Significant $\mathrm{P}$ values $(<0.05)$ were bolded

Table 2. Association between SNPs in DNL Pathway Related Genes and Overall Survival of HCC Patients

\begin{tabular}{|c|c|c|c|c|c|}
\hline Gene symbol & SNP & Predict function & Genetic Models & $\mathrm{HR}(95 \% \mathrm{CI})^{*}$ & $\mathrm{P}$ value \\
\hline \multirow[t]{6}{*}{ ACLY } & \multirow[t]{3}{*}{ rs2304497 } & \multirow[t]{3}{*}{ Missense } & Additive & $1.08(0.83-1.42)$ & 0.559 \\
\hline & & & Dominant & $1.08(0.82-1.43)$ & 0.565 \\
\hline & & & Recessive & $1.17(0.16-8.61)$ & 0.875 \\
\hline & \multirow[t]{3}{*}{ rs9912300 } & \multirow[t]{3}{*}{ TFBS } & Additive & $1.17(0.97-1.42)$ & 0.099 \\
\hline & & & Dominant & $1.19(0.95-1.49)$ & 0.141 \\
\hline & & & Recessive & $1.36(0.81-2.30)$ & 0.243 \\
\hline \multirow[t]{9}{*}{$\mathrm{ACACA}$} & \multirow[t]{3}{*}{ rs 1714987} & \multirow[t]{3}{*}{ Splicing } & Additive & $1.01(0.86-1.19)$ & 0.893 \\
\hline & & & Dominant & 0.93(0.73-1.19) & 0.553 \\
\hline & & & Recessive & $1.13(0.86-1.49)$ & 0.363 \\
\hline & \multirow[t]{3}{*}{ rs7211875 } & \multirow[t]{3}{*}{ Missense } & Additive & $0.95(0.78-1.16)$ & 0.635 \\
\hline & & & Dominant & $0.93(0.74-1.16)$ & 0.499 \\
\hline & & & Recessive & $1.13(0.63-2.02)$ & 0.688 \\
\hline & \multirow[t]{3}{*}{ rs11871275 } & \multirow[t]{3}{*}{ TFBS } & Additive & $1.13(0.92-1.39)$ & 0.231 \\
\hline & & & Dominant & $1.17(0.92-1.48)$ & 0.193 \\
\hline & & & Recessive & $1.07(0.54-2.09)$ & 0.850 \\
\hline \multirow[t]{12}{*}{ FASN } & \multirow[t]{3}{*}{ rs4246444 } & \multirow[t]{3}{*}{ NA } & Additive & $1.06(0.86-1.31)$ & 0.581 \\
\hline & & & Dominant & $1.23(0.91-1.67)$ & 0.173 \\
\hline & & & Recessive & $0.85(0.57-1.26)$ & 0.416 \\
\hline & \multirow[t]{3}{*}{ rs1140616 } & \multirow[t]{3}{*}{ Synomous } & Additive & $0.97(0.82-1.15)$ & 0.757 \\
\hline & & & Dominant & $0.96(0.77-1.20)$ & 0.746 \\
\hline & & & Recessive & $0.97(0.68-1.40)$ & 0.891 \\
\hline & \multirow[t]{3}{*}{ rs4485435 } & \multirow[t]{3}{*}{ Synomous } & Additive & $1.23(0.98-1.56)$ & 0.080 \\
\hline & & & Dominant & $1.22(0.95-1.58)$ & 0.124 \\
\hline & & & Recessive & $1.78(0.78-4.07)$ & 0.170 \\
\hline & \multirow[t]{3}{*}{ rs 11653012} & \multirow[t]{3}{*}{ TFBS } & Additive & $0.92(0.72-1.17)$ & 0.494 \\
\hline & & & Dominant & $0.92(0.71-1.18)$ & 0.502 \\
\hline & & & Recessive & $0.81(0.11-5.79)$ & 0.829 \\
\hline
\end{tabular}

*Abbreviations: ACLY, ATP citrate lyase; ACACA, Acetyl-CoA carboxylase 1; FASN, fatty acid synthase; CI, confidence interval; HR, hazard ratio; Ref., reference; TFBS, transcriptional factor binding site; NA, not available. *Adjusted for gender, age, serum HBsAg, serum AFP level, TNM stage, and treatments 
You-Sheng Wu et al

Table 3. Association between SNPs and Overall Survival of HCC Patients Stratified by AFP Level

\begin{tabular}{|c|c|c|c|c|c|c|}
\hline \multirow[t]{2}{*}{ Gene symbol } & \multirow[t]{2}{*}{ SNP } & \multirow[t]{2}{*}{ Genetic Models } & \multicolumn{2}{|c|}{ Patients with lower AFP $(<200 \mathrm{ng} / \mathrm{ml})$} & \multicolumn{2}{|c|}{ Patients with higher AFP $(\geq 200 \mathrm{ng} / \mathrm{ml})$} \\
\hline & & & $\mathrm{HR}(95 \% \mathrm{CI}) *$ & $P$ value & $\operatorname{HR}(95 \% \mathrm{CI})^{*}$ & $\mathrm{P}$ value \\
\hline \multirow[t]{6}{*}{ ACLY } & \multirow[t]{3}{*}{ rs2304497 } & Additive & $1.18(0.76-1.82)$ & 0.466 & $1.11(0.79-1.58)$ & 0.541 \\
\hline & & Dominant & $1.18(0.76-1.82)$ & 0.466 & $1.12(0.78-1.61)$ & 0.535 \\
\hline & & Recessive & NA & NA & $1.09(0.15-8.09)$ & 0.935 \\
\hline & \multirow[t]{3}{*}{ rs9912300 } & Additive & $1.38(1.03-1.85)$ & 0.032 & $1.11(0.86-1.43)$ & 0.425 \\
\hline & & Dominant & $1.45(1.01-2.08)$ & 0.046 & $1.12(0.83-1.51)$ & 0.461 \\
\hline & & Recessive & $1.69(0.77-3.70)$ & 0.189 & $1.19(0.58-2.44)$ & 0.627 \\
\hline \multirow[t]{9}{*}{ ACACA } & \multirow[t]{3}{*}{ rs 1714987} & Additive & $0.97(0.76-1.25)$ & 0.831 & $1.03(0.83-1.28)$ & 0.790 \\
\hline & & Dominant & $1.02(0.69-1.50)$ & 0.936 & $0.88(0.64-1.20)$ & 0.417 \\
\hline & & Recessive & $0.91(0.59-1.40)$ & 0.654 & $1.30(0.91-1.86)$ & 0.154 \\
\hline & \multirow[t]{3}{*}{ rs7211875 } & Additive & $1.02(0.75-1.38)$ & 0.917 & $0.87(0.66-1.14)$ & 0.317 \\
\hline & & Dominant & $0.96(0.68-1.35)$ & 0.799 & $0.87(0.64-1.17)$ & 0.343 \\
\hline & & Recessive & $1.50(0.68-3.31)$ & 0.314 & $0.80(0.32-2.01)$ & 0.637 \\
\hline & \multirow[t]{3}{*}{ rs 11871275} & Additive & $0.95(0.69-1.30)$ & 0.735 & $1.33(1.01-1.75)$ & 0.041 \\
\hline & & Dominant & $0.93(0.64-1.36)$ & 0.725 & $1.40(1.03-1.90)$ & 0.034 \\
\hline & & Recessive & $0.94(0.38-2.33)$ & 0.898 & $1.24(0.45-3.40)$ & 0.681 \\
\hline \multirow[t]{12}{*}{ FASN } & \multirow[t]{3}{*}{ rs4246444 } & Additive & $1.13(0.81-1.58)$ & 0.479 & $1.04(0.79-1.38)$ & 0.759 \\
\hline & & Dominant & $1.15(0.73-1.80)$ & 0.550 & $1.31(0.86-1.99)$ & 0.205 \\
\hline & & Recessive & $1.15(0.63-2.11)$ & 0.641 & $0.74(0.43-1.28)$ & 0.280 \\
\hline & \multirow[t]{3}{*}{ rs1140616 } & Additive & $1.09(0.84-1.40)$ & 0.524 & $0.91(0.72-1.14)$ & 0.419 \\
\hline & & Dominant & $1.08(0.76-1.53)$ & 0.662 & $0.92(0.68-1.23)$ & 0.558 \\
\hline & & Recessive & $1.19(0.71-1.99)$ & 0.510 & $0.81(0.48-1.38)$ & 0.441 \\
\hline & \multirow[t]{3}{*}{ rs4485435 } & Additive & $0.97(0.76-1.25)$ & 0.831 & $1.03(0.83-1.28)$ & 0.790 \\
\hline & & Dominant & $1.30(0.86-1.95)$ & 0.212 & $1.15(0.82-1.60)$ & 0.425 \\
\hline & & Recessive & $1.29(0.17-9.78)$ & 0.805 & $1.95(0.79-4.83)$ & 0.150 \\
\hline & \multirow[t]{3}{*}{ rs 11653012} & Additive & $0.88(0.59-1.32)$ & 0.541 & $0.95(0.69-1.30)$ & 0.758 \\
\hline & & Dominant & $0.89(0.59-1.33)$ & 0.564 & $0.95(0.69-1.32)$ & 0.766 \\
\hline & & Recessive & NA & NA & $0.88(0.12-6.44)$ & 0.903 \\
\hline
\end{tabular}

*Abbreviations: ACLY, ATP citrate lyase; ACACA, Acetyl-CoA carboxylase 1; FASN, fatty acid synthase; CI, confidence interval; HR, hazard ratio; Ref., reference; *Adjusted for gender, age, serum HBsAg status, TNM stage, and treatment; Significant $\mathrm{P}$ values $(<0.05)$ were bolded
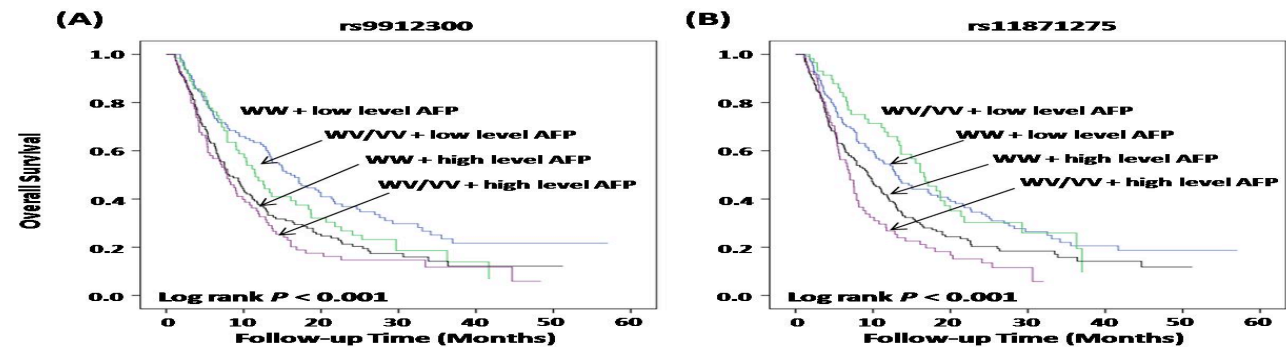

Figure 1. Kaplan-Meier Curve Analysis for the Join Effect of Serum AFP Level and SNP rs9912300 (A) or SNP rs11871275 Under Dominant Model. WW, homozygous wild genotype, WV/VV, variant-containing genotype. The curves corresponding to each patient group were indicated by arrows

Table 4. Joint Effect of SNPs and AFP Levels on HCC Overall Survival

\begin{tabular}{lrcc}
\hline Cvariables & Death/Total & HR $(95 \% \text { CI })^{*}$ & P value \\
\hline rs9912300 & & & \\
WW+lower level AFP & $86 / 126$ & Ref. & \\
WV/VV+lower level AFP & $50 / 63$ & $1.31(0.92-1.86)$ & 0.135 \\
WW+higher level AFP & $116 / 144$ & $1.46(1.10-1.95)$ & $\mathbf{0 . 0 0 9}$ \\
WV/VV+higher level AFP & $72 / 84$ & $1.62(1.17-2.24)$ & $\mathbf{0 . 0 0 4}$ \\
P for interaction & & & $\mathbf{0 . 0 0 2}$ \\
rs11871275 & & & \\
WW+lower level AFP & $96 / 131$ & Ref. & \\
WV/VV+lower level AFP & $40 / 58$ & $0.89(0.62-1.30)$ & 0.552 \\
WW+higher level AFP & $126 / 158$ & $1.22(0.93-1.61)$ & 0.156 \\
WV/VV+higher level AFP & $63 / 71$ & $1.74(1.25-2.42)$ & $\mathbf{0 . 0 0 1}$ \\
P for interaction & & & $\mathbf{0 . 0 0 2}$
\end{tabular}

Abbreviations: CI, confidence interval; HR, hazard ratio; Ref., reference; *Adjusted for gender, age, serum HBsAg status, TNM stage, and treatment; Significant $\mathrm{P}$ values $(<0.05)$ were bolded
95\% CI 1.03-1.85, $p=0.032)$ and dominant $(\mathrm{HR}=1.45$, 95\%CI 1.01-2.08, $p=0.046)$ models, and SNP rs11871275 in ACACA gene exhibited significant associations with overall survival in HCC patients with higher serum AFP $(\geq 200 \mathrm{ng} / \mathrm{ml})$ under additive $(\mathrm{HR}=1.33,95 \% \mathrm{CI} 1.01-1.75$, $p=0.041)$ and dominant $(\mathrm{HR}=1.40,95 \% \mathrm{CI} 1.03-1.90$, $p=0.034$ ) models (Table 3 ).

Joint effect of significant SNPs and serum AFP levels on HCC overall survival

Since the significant association result between SNPs and cancer outcomes was observed in HCC patients with different serum AFP levels. To reveal if there is an interaction between SNPs and serum AFP level, we conducted joint analysis of the two significant SNPs and AFP level on HCC outcome with adjustment for gender, age, serum HBsAg status, TNM stage, and treatment 
under dominant model. As data shown in Table 4, in both SNP rs9912300 and rs11871275, patients with variantcontaining genotypes and higher level of serum AFP exhibited significant increased death risk with $\mathrm{HR}$ of 1.62 (95\% CI 1.17-2.24, $p=0.004)$ and 1.74 (95\% CI 1.25-2.42, $p=0.001)$, respectively, comparing to those with wild genotype and lower serum AFP level. Furthermore, the effects on HCC patients by combination of SNP and AFP level showed statistically significant interaction with $p$ for interaction of 0.002 for both SNPs. In consist with the Cox proportional hazard analysis, Kaplan-Meier curves analysis also showed that patients higher risk genotypes (variant-containing genotypes in both SNPs) and higher serum AFP level had significantly shorter overall survival time with log-rank $p<0.001$ in both SNPs, when comparing those with lower risk genotype (wild genotypes in both SNPs) and lower serum AFP level (Figure 1).

\section{Discussion}

In the current study, we evaluated the effects of nine functional SNPs in three genes (ACLY ACACA, and FASN) of DNL pathway on the prognosis of HCC patients treated with TACE. In the stratified analysis, we found that SNP rs9912300 in ACLY gene and SNP rs11871275 in ACACA gene were significantly associated with overall survival of HCC patients with lower and higher serum AFP levels, respectively. Furthermore, joint analysis results suggested that there were significant interaction between these two SNPs and serum AFP level on HCC outcome. To the best of our knowledge, this is the first study to report that genetic variants in three genes of DNL pathway had significant associations with overall survival of Chinese HCC patients treated by TACE.

Reprogramming of cellular metabolism is a hallmark of cancer. In most normal cells, de novo fatty acid synthesis is usually suppressed, however, upregulated de novo fatty acid synthesis has been widely observed in cancer cells, which fuels membrane biogenesis in rapidly proliferating cancer cells and renders membrane lipid more saturated, thereby plays an important role in tumorigenesis and tumor progression (Rysman et al., 2010; Hanahan and Weinberg, 2011). The importance of the lipogenic enzymes in survival, proliferation and progression of cancer cells have been well recognized (Swinnen et al., 2006). ACLY catalyzes the conversion of mitochondria-derived citrate to cytosolic acetyl-CoA, which is a vital precursor for the endogenous biosynthesis of fatty acids and cholesterol, and is involved isoprenoid-based protein modification (Hatzivassiliou et al., 2005; Zaidi et al., 2012b). Increased ACLY levels and/or activity had been observed in various cancers, including HCC. Moreover, the overexpression of ACLY has been reported to be significantly associated with poor prognosis in cancers (Zhou et al., 2013; Lettieri Barbato et al., 2014). Similarly to ACLY, ACACAas another core enzyme involving in de novo fatty acid synthesis has also been found to be upretulated in various proliferating cancer cells (Abu-Elheiga et al., 2001; Chajes et al., 2006). Although the molecular mechanisms of ACLY and ACACA upregulation in cancer cells are still not determined, these data suggest that expression of these enzymes could be a potential marker for predicting cancer prognosis. In this study, we identified that SNPs rs 9912300 and rs 11871275 were significantly associated with poor prognosis in a subgroup of HCC patients treated by TACE. Since these two SNPs are located in the predicted transcriptional factor binding sites of ACLY and ACACA genes, they are possible to alter the expressions of ACLY and ACACA enzymes. The underlying mechanisms of rs9912300 and rs11871275 involved in HCC prognosis would be our further research aim.

Genetic variants such as SNPs may affect the expression and functions of genes. Considering the important role of DNL genes in tumor progression, it is necessary to investigate SNPs effects in these genes on cancer cell proliferation, drug sensitivity as well as clinicopathologcal characteristics and prognosis. Until now, there are few researches involving the relationships between these SNPs and cancer patient prognosis. It has been reported that SNP rs9912300 in ACLY gene is significantly associated with the overall survival of NSCLC patients under dominant model (Jin et al., 2014), which is in concordance with our finding that rs 9912300 was significantly associated with overall survival of TACE-treated HCC patients. Additionally, it has been reported that SNP rs1127678 in FASN gene is associated with the risk and mortality of prostate cancer (Nguyen et al., 2010). SNPs rs4246444 and rs4485435 in FASN gene has been found to be significantly associated with the risk of recurrence in NSCLC patients (Jin et al., 2014). However, we did not identify significant associations between SNPs in FASN gene and HCC outcomes in either overall or stratified analysis. These data suggest that the effect on cancer patient outcomes conferred by SNPs in DNL pathway genes might dependent on specific cancer type.

AFP was first identified as a serum glycoprotein produced by the yolk sac endoderm and the liver during normal fetal development, and its expression level drops dramatically after birth (Bergstrand and Czar, 1956). Pathologically, AFP is produced and released into the circulation when liver injured or HCC developed, and serum AFP level together with other clinical parameters is widely used as a biomarker for HCC diagnosis or prognosis prediction (Sell, 2008). In this study, we observed that SNP rs9912300 in ACLY gene and SNP rs 11871275 in ACACA gene were significantly associated with overall survival of TACE treated HCC patients with lower AFP level and higher AFP level under dominant model, respectively. Further analysis indicated that both SNPs had statistically significant interaction with serum AFP level in HCC patients. Although there is no mechanism study to support how AFP functionally interacted with ACLY or ACACA enzymes in de novo lipid synthesis during HCC development, the combined effects of AFP and polymorphisms in ACLY or ACACA gene had potential clinical significance in HCC tailored treatment, if our data was further validated.

Conclusively, our data strongly indicated that genetic variants of ACLY and ACACA genes may be independent prognostic biomarkers for overall survival prediction in a hospital-base cohort of HCC patients treated by TACE. 
Our findings contribute to the current understanding on the functional roles of polymorphisms in DNL pathway genes in clinical outcome of HCC patients after TACE treatment.

\section{References}

Abramson HN (2011). The lipogenesis pathway as a cancer target. J Med Chem, 54, 5615-38.

Abu-Elheiga L, Matzuk MM, Abo-Hashema KA, et al (2001). Continuous fatty acid oxidation and reduced fat storage in mice lacking acetyl-CoA carboxylase 2. Science, 291, 2613-6.

Bergstrand CG, Czar B (1956). Demonstration of a new protein fraction in serum from the human fetus. Scand J Clin Lab Invest, $\mathbf{8}, 174$.

Bruix J, Sherman M (2011). Management of hepatocellular carcinoma: an update. Hepatology, 53, 1020-2.

Calvisi DF, Wang C, Ho C, et al (2011). Increased lipogenesis, induced by AKT-mTORC1-RPS6 signaling, promotes development of human hepatocellular carcinoma. Gastroenterology, 140, 1071-83.

Chajes V, Cambot M, Moreau K, et al (2006). Acetyl-CoA carboxylase alpha is essential to breast cancer cell survival. Cancer Res, 66, 5287-94.

Chamary JV, Parmley JL, Hurst LD (2006). Hearing silence: non-neutral evolution at synonymous sites in mammals. Nat Rev Genet, 7, 98-108.

Dorn C, Riener MO, Kirovski G, et al (2010). Expression of fatty acid synthase in nonalcoholic fatty liver disease. Int $J$ Clin Exp Pathol, 3, 505-14.

Eggert SL, Huyck KL, Somasundaram P, et al (2012). Genomewide linkage and association analyses implicate FASN in predisposition to uterine leiomyomata. Am J Hum Genet, 91, 621-8.

Furuta E, Okuda H, Kobayashi A, et al (2010). Metabolic genes in cancer: their roles in tumor progression and clinical implications. Biochim Biophys Acta, 1805, 141-52.

Hanahan D, Weinberg RA (2011). Hallmarks of cancer: the next generation. Cell, 144, 646-74.

Hatzivassiliou G, Zhao F, Bauer DE, et al (2005). ATP citrate lyase inhibition can suppress tumor cell growth. Cancer Cell, 8, 311-21.

Jemal A, Bray F, Center MM, et al (2011). Global cancer statistics. CA Cancer J Clin, 61, 69-90.

Jemal A, Siegel R, Xu J, et al (2010). Cancer statistics, 2010. CA Cancer J Clin, 60, 277-300.

Jin X, Zhang KJ, Guo X, et al (2014). Fatty Acid synthesis pathway genetic variants and clinical outcome of non-small cell lung cancer patients after surgery. Asian Pac J Cancer Prev, 15, 7097-103.

Laing RE, Hess P, Shen Y, et al (2011). The role and impact of SNPs in pharmacogenomics and personalized medicine. Curr Drug Metab, 12, 460-86.

Lettieri Barbato D, Vegliante R, Desideri E, et al (2014). Managing lipid metabolism in proliferating cells: new perspective for metformin usage in cancer therapy. Biochim Biophys Acta, 1845, 317-24.

Lin DY, Lin SM, Liaw YF (1997). Non-surgical treatment of hepatocellular carcinoma. J Gastroenterol Hepatol, 12, 319-28.

Liu HB, Peng YP, Dou CW, et al (2012). Comprehensive study on associations between nine SNPs and glioma risk. Asian Pac J Cancer Prev, 13, 4905-8.

Llovet JM, Bruix J (2003). Systematic review of randomized trials for unresectable hepatocellular carcinoma: Chemoembolization improves survival. Hepatology, 37, 429-42.
Llovet JM, Real MI, Montana X, et al (2002). Arterial embolisation or chemoembolisation versus symptomatic treatment in patients with unresectable hepatocellular carcinoma: a randomised controlled trial. Lancet, 359, 1734-9.

Mao J, DeMayo FJ, Li H, et al (2006). Liver-specific deletion of acetyl-CoA carboxylase 1 reduces hepatic triglyceride accumulation without affecting glucose homeostasis. Proc Natl Acad Sci USA, 103, 8552-7.

Mashima T, Seimiya H, Tsuruo T (2009). De novo fatty-acid synthesis and related pathways as molecular targets for cancer therapy. Br J Cancer, 100, 1369-72.

Menendez JA, Lupu R (2007). Fatty acid synthase and the lipogenic phenotype in cancer pathogenesis. Nat Rev Cancer, 7, 763-77.

Migita T, Ruiz S, Fornari A, et al (2009). Fatty acid synthase: a metabolic enzyme and candidate oncogene in prostate cancer. J Natl Cancer Inst, 101, 519-32.

Nguyen PL, Ma J, Chavarro JE, et al (2010). Fatty acid synthase polymorphisms, tumor expression, body mass index, prostate cancer risk, and survival. J Clin Oncol, 28, 3958-64.

Rysman E, Brusselmans K, Scheys K, et al (2010). De novo lipogenesis protects cancer cells from free radicals and chemotherapeutics by promoting membrane lipid saturation. Cancer Res, 70, 8117-26.

Sell S (2008). Alpha-fetoprotein, stem cells and cancer: how study of the production of alpha-fetoprotein during chemical hepatocarcinogenesis led to reaffirmation of the stem cell theory of cancer. Tumour Biol, 29, 161-80.

Swinnen JV, Brusselmans K, Verhoeven G (2006). Increased lipogenesis in cancer cells: new players, novel targets. Curr Opin Clin Nutr Metab Care, 9, 358-65.

Watson JA, Fang M, Lowenstein JM (1969). Tricarballylate and hydroxycitrate: substrate and inhibitor of ATP: citrate oxaloacetate lyase. Arch Biochem Biophys, 135, 209-17.

Xing J, Myers RE, He X, et al (2011). GWAS-identified colorectal cancer susceptibility locus associates with disease prognosis. Eur J Cancer, 47, 1699-707.

Yoon S, Lee MY, Park SW, et al (2007). Up-regulation of acetylCoA carboxylase alpha and fatty acid synthase by human epidermal growth factor receptor 2 at the translational level in breast cancer cells. J Biol Chem, 282, 26122-31.

Yuan P, Wang S, Zhou F, et al (2014). Functional polymorphisms in the NPAS2 gene are associated with overall survival in transcatheter arterial chemoembolization-treated hepatocellular carcinoma patients. Cancer Sci, 105, 825-32.

Zaidi N, Royaux I, Swinnen JV, et al (2012a). ATP citrate lyase knockdown induces growth arrest and apoptosis through different cell- and environment-dependent mechanisms. Mol Cancer Ther, 11, 1925-35.

Zaidi N, Swinnen JV, Smans K (2012b). ATP-citrate lyase: a key player in cancer metabolism. Cancer Res, 72, 3709-14.

Zhou F, He X, Liu H, et al (2012). Functional polymorphisms of circadian positive feedback regulation genes and clinical outcome of Chinese patients with resected colorectal cancer. Cancer, 118, 937-46.

Zhou Y, Bollu LR, Tozzi F, et al (2013). ATP citrate lyase mediates resistance of colorectal cancer cells to SN38. Mol Cancer Ther, 12, 2782-91. 